\title{
Antipsychotic use in dementia patients in a general practice setting: a Dutch population-based study
}

Received 2 January 2016; Accepted 18 February 2016; First published online 18 March 2016

Key words: Antipsychotics, dementia, healthcare database, Netherlands.

\section{Introduction}

Antipsychotic (AP) prescribing in elderly persons has been a focus of attention from drug regulatory agencies in the past decade. These drugs are commonly prescribed off-label for behavioural and psychological symptoms in dementia (BPSD). Risperidone is specifically approved for persistent aggression in patients with moderate to severe Alzheimer's dementia for up to 6 weeks but other commonly used APs such as olanzapine, quetiapine and haloperidol do not have a specific indication for dementia-related symptoms. The lack of more effective pharmacological options has led to a widespread overuse of APs in dementia, including specific drugs such as quetiapine for which there is very limited evidence supporting its efficacy in BPSD based on clinical trial data (Ballard \& Waite, 2006).

In 2004, the European Medicines Agency (EMA) launched a first safety warning informing healthcare providers that the use of olanzapine and risperidone was associated with an increased risk of stroke as well as all-cause mortality (European Medicines Agency, 2004) and by August 2009 EMA extended the warning to all AP use in dementia (European Medicines Agency, 2009). Observational studies investigating AP use in dementia within Europe suggest that international and national safety warnings may have had only a short-term impact on AP prescribing (Sanfélix-Gimeno et al. 2009; Trifirò et al. 2010b; Franchi et al. 2012; Guthrie et al. 2013; Schulze et al. 2013; Gallini et al. 2014). Such warnings may also have prompted the use of other APs, replacing those used previously, rather than reducing the excess use of these drugs.

In the Netherlands, recent investigations of AP use in older persons has focused on institutionalised elderly persons (Sterke et al. 2012; van de ven-Vakhteva

\footnotetext{
* Address for correspondence: G. Trifirò, Department of Biomedical and Dental Sciences and Morpho-functional Imaging, University of Messina, Via Consolare Valeria, 98125, Messina, Italy.

(Email: trifirog@unime.it)
}

et al. 2013; van der Speck et al. 2013; Kleijer et al. 2014; van der Putten et al. 2014). The prevalence of AP use in community-dwelling Dutch elderly persons with dementia in recent years has not been estimated. Nevertheless, two nested case-control studies using the Dutch Integrated Primary Care Information (IPCI) database suggest that both atypical and conventional APs are associated with increased risk of death and community-acquired pneumonia in elderly persons (Trifirò et al. 2007, 2010a) and a case-control study using the Dutch PHARMO record linkage system found an increased risk of cerebrovascular events in elderly persons (Kleijer et al. 2009). The aim of this populationbased study was therefore to explore whether the prevalence of AP use changed in a cohort of dementia patients in a Dutch general practice database after the warning launched by EMA in August 2009.

\section{Methods}

\section{Data source}

The IPCI database is a Dutch general practice database containing complete electronic health records from 466 general practices. There are around 1786000 patients registered in IPCI, who are representative of the Dutch general population in terms of age and sex distribution. Available data include medical diagnoses, coded using International Classification of Primary Care codes, prescription data, coded using anatomical therapeutic chemical (ATC) classification system and additional medical information (e.g., laboratory measurements, functionality status variables, etc.) among others, as well free text clinical notes. IPCI has been used extensively for pharmacoepidemiology research (Straus et al. 2004; Trifirò et al. 2007, 2010a, b).

\section{Population}

Persons in IPCI were considered eligible if they had a minimum 1 year of database history, were alive and aged 65 years or older and had a diagnosis of dementia 
over the observation period 1st January 2008-31st December 2013. Patient contribution to the study was censored at the end of study period, i.e., 31st December 2013, transfer out of database/end of registration in IPCI or death.

\section{Exposure}

Prescriptions of APs were identified using ATC codes: N05A (except for N05AN which corresponds to lithium). The APs identified were grouped by class as atypical (ATC: N05AX08, N05AX11, N05AX12, N05AX13, N05AX14, N05AE03, N05AE04, N05AE05, N05AH02, N05AH03, N05AH04, N05AH05, N05AL05) and conventional (all others N05A, except for N05AN). The prevalence of the commonly used APs risperidone, quetiapine, olanzapine and haloperidol were investigated separately.

\section{Analysis}

The trimester prevalence of AP use was calculated dividing the number of persons receiving at least one AP prescription (numerator) by the number of persons registered in the database in the same trimester. Data management and analysis were carried out using SAS Release 9.3 (SAS Institute, Cary, NC, USA).

\section{Review board approval}

The study was approved by IPCI Review Board (IPCI Raad van Toezicht).

\section{Results}

From 2008 to 2013314191 patients aged 65 and over were identified in IPCI. Of these, 14396 (4.6\%) had a diagnosis of dementia. At the start of the observation period (2008), the trimester prevalence of any AP use was $13 \%$ in elderly dementia patients, decreasing to $11 \%$ just before the warning in the third trimester of 2009 (Fig. 1). The use of any APs increased mildly in the 3 months after the warning (from 10 to $11 \%$ ), thereafter decreasing gradually to $8 \%$. The trend for conventional APs was very similar to that for APs overall over the observation period, starting at $10 \%$ in 2008 decreasing to $7 \%$ in the second trimester of 2009 . There was briefly a small increase in prevalence from 6 to $8 \% 3$ months after the warning, after which there was a general decrease to $7 \%$ at the end of the observation period. The trimester prevalence of atypical APs was much lower, at approximately $4 \%$ throughout the prewarning period; this initially decreased from 4 to $3 \%$ over the year after the warning, and remained stable, fluctuating between 3 and $4 \%$. Haloperidol was the most commonly used AP. The prevalence of haloperidol fluctuated between 4 and 5\% before the warning and initially rose slightly from 5 to $6 \%$ in the trimester after the warning, after which there was a gradual decrease to $4 \%$. The second most commonly used AP was risperidone, with a prevalence of $2-3 \%$ throughout the observation period. Quetiapine and olanzapine had a very low prevalence of use, remaining stable at $1 \%$ throughout the observation period.

\section{Discussion}

The most commonly prescribed APs among elderly patients with dementia in Dutch general practice between 2008 and 2013 were haloperidol and risperidone. This is in line with Dutch clinical guidelines for General Practitioners for dementia (Dutch College of General Practicioners, 2015) suggesting that haloperidol and risperidone can be used in cases of acute psychosis and/or aggression if non-pharmacological approaches are not successful. The low prevalence of quetiapine (approximately $1 \%$ during the whole study period) is consistent with the less convincing evidence supporting the efficacy of this drug in BPSD. In other countries, quetiapine was found to be prescribed in older people with dementia more frequently than other AP drugs (Franchi et al. 2012; Guthrie et al. 2013). In their study carried out using the Lombardy Administrative Database (Italy) from 2002 to 2008, Franchi et al. found that by 2008, quetiapine was by far the most commonly prescribed drug in elderly persons taking anti-cholinesterase inhibitors (a proxy of dementia), with an annual prevalence of $12 \%$; to put this in context, this prevalence could be compared that of the next most commonly prescribed AP in Lombardy, haloperidol, of $3 \%$ in 2008 . The quarterly prevalence of use of quetiapine in Scottish persons with dementia peaked to $10 \%$ in 2010 but gradually decreased to $6 \%$ by 2011 (Guthrie et al. 2013).

The higher use of conventional agents rather than atypical ones in dementia is of clinical significance because randomised clinical trials investigating the effectiveness of APs did not find evidence favouring the use of conventional APs in dementia. On the other hand, such trials did find a modest improvement in aggression and smaller but nevertheless significant benefit in psychosis over 6-12 weeks of treatment with risperidone and olanzapine (Ballard \& Waite, 2006). After the 2009 EMA warning, the use of both classes of drugs continued to decline.

The elevated use of conventional AP use in Dutch persons with dementia (6\% quarterly prevalence by the end of 2013) compared with atypical APs (4\% quarterly prevalence by the end of 2013) was also seen in dementia patients in France and 


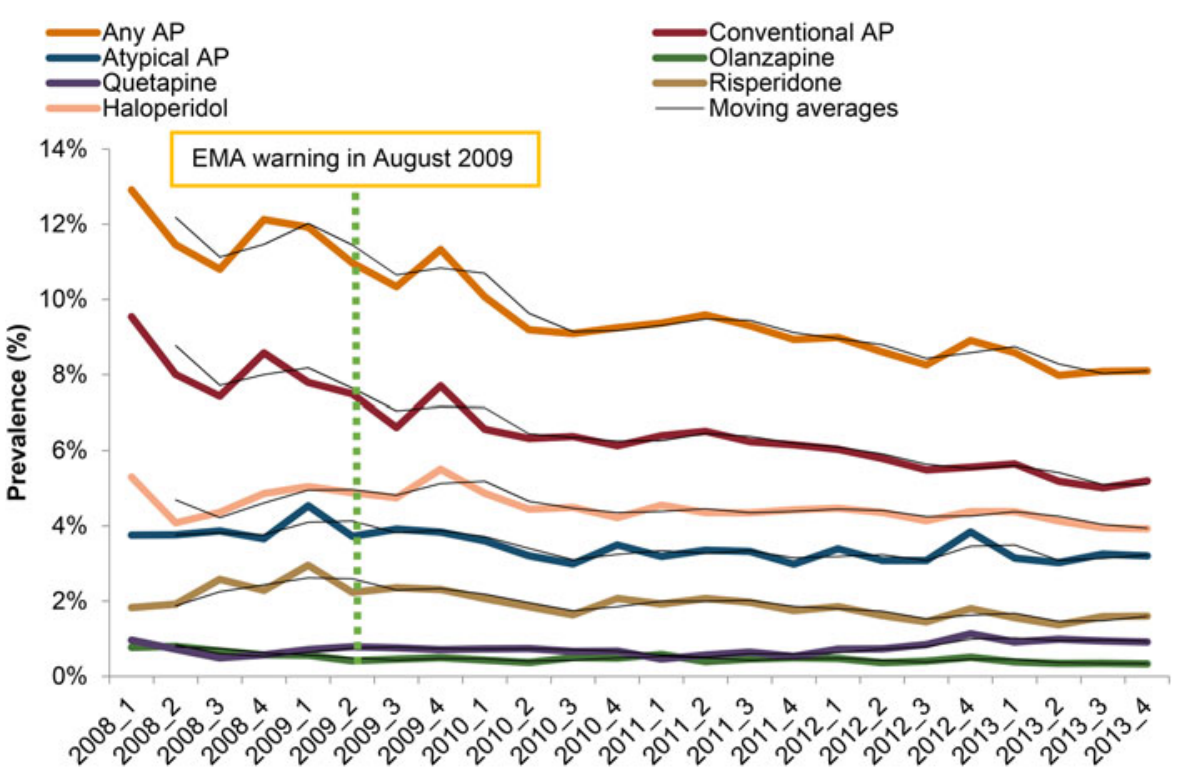

Fig. 1. Prevalence of antipsychotic use in elderly dementia patients in a Dutch general practice setting. AP, antipsychotics.

Germany (Schulze et al. 2013; Gallini et al. 2014). In French community-dwelling persons $>65$ with dementia, the monthly prevalence of conventional AP use was $15 \%$ in 2003 compared with $4 \%$ for atypical AP use (Gallini et al. 2014). However by 2012, both classes of drugs had a similar monthly prevalence at approximately $5 \%$. In Germany, the annual prevalence of conventional AP use among persons with dementia estimated using health insurance data decreased from $35 \%$ in 2004 to $32 \%$ in 2009 , but remained higher than atypical AP use which increased marginally from $17 \%$ in 2004 to $20 \%$ in 2009 (Schulze et al. 2013). In Italy, on the other hand, atypical APs were more commonly used than conventional ones in persons with dementia, with an annual prevalence of $15 \%$ in 2008 compared with 5\% for conventional APs (Franchi et al. 2012). This is most likely explained by the very high use of quetiapine in this population as described above.

The higher use of conventional APs in some countries may have been at least partly prompted by drug safety warnings which initially cautioned prescribers regarding the increased risk of all-cause mortality and stroke associated with olanzapine and risperidone in 2004 (European Medicines Agency, 2004), although national and international drug regulatory agencies extended the warning to all AP use in dementia in 2009 (European Medicines Agency, 2009).

The use of APs overall in the Dutch community setting at the end of the observation period (quarterly prevalence of approximately $9 \%$ in 2013) was much lower compared with the use of these drugs in the longterm care setting. A recent study found that $32 \%$ of a sample of 290 Dutch long-term care residents was prescribed an AP (van der Putten et al. 2014). Similar findings were reported in the same year in a larger study ( $N=1090$ Dutch long-term care residents from 20 long-term care residences), where 31\% long-term care residents were prescribed at least one AP drug (Kleijer et al. 2014).

Further information is needed to understand whether the changes in AP use in dementia correspond to increase in the appropriateness of drug prescribing. Strategic action is required to promote the appropriate use of APs in dementia patients and facilitate the adoption of non-pharmacological treatment, the latter currently being underutilised for reasons that include low awareness among healthcare professionals of their efficacy and implementation as well as the nonreimbursable status of non-pharmacological interventions (Kales et al. 2014).

\section{Acknowledgement}

None.

\section{Financial Support}

This study received funding from the Italian Health Ministry in the context of the Project 'GR-20091607316 - Assessment of the safety of AP drugs in elderly with dementia: an international, population-based study using healthcare databases'.

\section{Conflict of Interest}

None of the authors has conflicts of interest directly related to the study. MS is heading a research unit that holds unconditional research contracts with some pharmaceutical companies (Eli Lilly, Pfizer, 
AstraZeneca Novartis, Boehringer, GSK, Servier), and none of them is related to this study.

J. Sultana ${ }^{\mathbf{1 , 2}}$, I. Leal ${ }^{\mathbf{2}}$, M. de Ridder ${ }^{2}$,
M. Sturkenboom ${ }^{\mathbf{2}}$ and G. Trifiro' ${ }^{\mathbf{1} \mathbf{3}^{*}}$
${ }^{1}$ Department of Clinical and Experimental Medicine, Via
Consolare Valeria, 98125, University of Messina,
Messina, Italy
${ }^{2}$ Department of Epidemiology, Erasmus Medical Centre,
Dr Molewaterplein 50, 3015 GE Rotterdam,
The Netherlands
${ }^{3}$ Department of Biomedical and Dental Sciences and
Morpho-functional Imaging, Via Consolare Valeria,
University of Messina, 98125 Messina, Italy

\section{References}

Ballard C, Waite J (2006). The effectiveness of atypical antipsychotics for the treatment of aggression and psychosis in Alzheimer's disease. Cochrane Database of Systematic Reviews CD003476.

Dutch College of General Practitioners (2015). Dementia: Summary Map. Retrieved 3 November 2015 from https:// www.nhg.org/standaarden/samenvatting/dementie

European Medicines Agency (EMA) (2004). EMEA public statement on the safety of olanzapine (Zyprexa, Zyprexa Velotab); London. Retrieved 24 November 2015 from http:// www.bfarm.de/SharedDocs/Downloads/DE/Arzneimittel/ Pharmakovigilanz/Service/aktuelles/emea-zyprexa-st.pdf? _blob=publicationFile\&v $=2$

European Medicines Agency (EMA) (2009). EMEA 2010 Priorities for Drug Safety Research: Safety aspects of antipsychotics in demented patients; London. Retrieved 24 November 2015 from http://www.ema.europa.eu/docs/ en_GB/document_library/Other/2010/03/WC500076323.pdf

Franchi C, Tettamanti M, Marengoni A, Bonometti F, Pasina L, Cortesi L, Fortino I, Bortolotti A, Merlino L, Lucca U, Riva E, Nobili A (2012). Changes in trend of antipsychotics prescription in patients treated with cholinesterase inhibitors after warnings from Italian Medicines Agency. Results from the EPIFARM-Elderly Project. European Journal of Neuropsychopharmacology 22, 569-577.

Gallini A, Andrieu S, Donohue JM, Oumouhou N, Lapeyre-Mestre M, Gardette V (2014). Trends in use of antipsychotics in elderly patients with dementia: impact of national safety warnings. European Journal of Neuropsychopharmacology 24, 95-104.

Guthrie B, Clark SA, Reynish EL, McCowan C, Morales D (2013). Differential impact of two risk communications on antipsychotic prescribing to people with dementia in Scotland: segmented regression time series analysis 20012011. PLOS ONE 8, e68976.

Kales HC, Gitlin LN, Lyketsos CG; Detroit Expert Panel on Assessment and Management of Neuropsychiatric Symptoms of Dementia (2014). Management of neuropsychiatric symptoms of dementia in clinical settings: recommendations from a multidisciplinary expert panel. Journal of the American Geriatriatric Society 62, 762-769.
Kleijer BC, van Marum RJ, Egberts AC, Jansen PA, Knol W, Heerdink ER (2009). Risk of cerebrovascular events in elderly users of antipsychotics. Journal of Psychopharmacology 23, 909-914.

Kleijer BC, van Marum RJ, Frijters DH, Jansen PA, Ribbe MW, Egberts AC, Heerdink ER (2014). Variability between nursing homes in prevalence of antipsychotic use in patients with dementia. International Psychogeriatriatrics 26, 363-371.

Sanfélix-Gimeno G, Cervera-Casino P, Peiró S, López-Valcarcel BG, Blázquez A, Barbera T (2009). Effectiveness of safety warnings in atypical antipsychotic drugs: an interrupted time-series analysis in Spain. Drug Safety 32, 1075-1087.

Schulze J, van den Bussche H, Glaeske G, Kaduszkiewicz H, Wiese B, Hoffmann F (2013). Impact of safety warnings on antipsychotic prescriptions in dementia: nothing has changed but the years and the substances. European Journal of Neuropsychopharmacology 23, 1034-1042.

Sterke CS, van Beeck EF, van der Velde N, Ziere G, Petrovic M, Looman CW, van der Cammen TJ (2012). New insights: dose-response relationship between psychotropic drugs and falls: a study in nursing home residents with dementia. Journal of Clinical Pharmacology 52, 947-955.

Straus SM, Bleumink GS, Dieleman JP, van der Lei J, 't Jong GW, Kingma JH, Sturkenboom MC, Stricker BH (2004). Antipsychotics and the risk of sudden cardiac death. Archives of Internal Medicine 164, 1293-1297.

Trifirò G, Verhamme KM, Ziere G, Caputi AP, Ch Stricker BH, Sturkenboom MC (2007). All-cause mortality associated with atypical and typical antipsychotics in demented outpatients. Pharmacoepidemiology and Drug Safety 16, 538-544.

Trifirò G, Gambassi G, Sen EF, Caputi AP, Bagnardi V, Brea J, Sturkenboom MC (2010a). Association of community-acquired pneumonia with antipsychotic drug use in elderly patients: a nested case-control study. Annals of Internal Medicine 152, 418-425, W139-W140.

Trifirò G, Sini G, Sturkenboom MC, Vanacore N, Mazzaglia G, Caputi AP, Cricelli C, Brignoli O, Aguglia E, Biggio G, Samani F (2010b). Prescribing pattern of antipsychotic drugs in the Italian general population 20002005: a focus on elderly with dementia. International Clinical Psychopharmacology 25, 22-28.

van de Ven-Vakhteeva J, Bor H, Wetzels RB, Koopmans RT, Zuidema SU (2013). The impact of antipsychotics and neuropsychiatric symptoms on the quality of life of people with dementia living in nursing homes. International Journal of Geriatric Psychiatry 28, 530-538.

van der Putten MJ, Wetzels RB, Bor H, Zuidema SU, Koopmans RT (2014). Antipsychotic drug prescription rates among Dutch nursing homes: the influence of patient characteristics and the dementia special care unit. Aging and Mental Health 18, 828-832.

van der Spek K, Gerritsen DL, Smalbrugge $M$, Nelissen-Vrancken MH, Wetzels RB, Smeets $\mathrm{CH}$, Zuidema SU, Koopmans RT (2013). PROPER I: frequency and appropriateness of psychotropic drugs use in nursing home patients and its associations: a study protocol. BMC Psychiatry 13, 307. 